\title{
Uso de MODELAÇÃo MATEMÁtICA PARA PROJETO dE CÂMARAS MECANIZADAS DE FLOCULAÇÃO EM SÉRIE EM ESTAÇõES DE TRATAMENTO DE ÁGUA
}

\author{
THE USE OF MATHEMATICAL MODELING TO THE DESIGN OF CONTINOUS \\ FLOW COMPLETELY MIXED CHAMBERS IN SERIES FOR FLOCCULATION IN \\ WATER TREATMENT PLANTS
}

\section{LUIZ DI BERNARDO}

Engenheiro Civil. Doutor em Engenharia Civil da Escola de Engenharia de São Carlos - USP. Professor Titular do Departamento de Hidráulica e Saneamento. Escola de Engenharia de São Carlos - USP

\section{ALEXANDRE BOTARI}

Engenheiro Civil. Doutorando em Engenharia Civil - Área de Concentração: Hidráulica e Saneamento. Escola de Engenharia de São Carlos - USP

\section{LYDA PATRICIA SABOGAL-PAZ}

Engenheira Sanitária. Doutoranda em Engenharia Civil - Área de Concentração: Hidráulica e Saneamento. Escola de Engenharia de São Carlos - USP

\author{
Recebido: 14/06/04 Aceito: 17/11/04
}

\section{RESUMO}

A metodologia encontrada na literatura para a otimização do gradiente de velocidade médio em reatores de mistura completa, com câmaras em série de escoamento contínuo, a partir de ensaios em reator estático tipo jarteste, baseia-se em modelos matemáticos que descrevem a cinética dos encontros entre partículas durante a floculação. Considerando-se que a aplicabilidade desta metodologia tem-se revelado inconsistente, como observado por diversos autores, este trabalho propõe uma metodologia racional para utilizar e otimizar os dados experimentais obtidos em reatores estáticos e, utilizando modelos matemáticos, obter dados para o projeto de floculadores mecanizados em série em estações de tratamento de água.

PALAVRAS-CHAVE: Gradiente de velocidade médio, modelação matemática, tempo de floculação, velocidade de sedimentação e floculação mecanizada em série.

\section{INTRODUÇÃO}

A realização de ensaios de laboratório e/ou construção de instalaçôes piloto é importante para a obtenção de parâmetros de projeto, principalmente em estações de tratamento de água que utilizam coagulantes químicos, uma vez que as condiçôes ótimas de coagulação, floculação, sedimentação e filtração estão diretamente associadas com a qualidade da água a ser tratada.

As particularidades inerentes ao processo de coagulação e à operação de floculação indicaram aos engenheiros a necessidade da realização de ensaios de laboratório, tais como aqueles feitos em equipamentos de jarteste, visando obter dados experimentais para projetos das Estações de Tratamento de Âgua - ETAs.

Os resultados obtidos em jarteste são, na maioria dos casos, utilizados sem se considerar o fator de escala, que é crítico quando se trabalha em reatores de mistura completa, principalmente nas câmaras de floculação mecanizadas em série. Portanto, obter o gradiente de velocidade médio e o tempo de floculação depende tanto dos resultados de laboratório, como da aplicação de metodologia apropriada para a elaboração do projeto.

Desta forma, o objetivo do presente trabalho é determinar o gradiente de velocidade médio e o tempo de floculação para a unidade de mistura completa de escoamento contínuo com câmaras em série, a partir dos resultados de ensaios em jarteste. Será aplicada a modelação matemática para estimar o desempenho em escala real da unidade de floculação utilizando cloreto férrico como coagulante no mecanismo de varredura. 


\section{Modelação matemática da floculação}

A floculação nas ETAs corresponde à etapa em que são fornecidas condições para facilitar o contato e a agregação de partículas previamente coaguladas, visando a formação de flocos com tamanho e massa específica que favoreçam sua remoção por sedimentação, flotação ou filtração rápida.

O desempenho da unidade de floculação depende da eficiência da unidade de mistura rápida, a qual é influenciada pelos seguintes fatores: tipo de coagulante, $\mathrm{pH}$ de coagulação, temperatura da água, concentração e idade da solução de coagulante, tempo e gradiente de velocidade da mistura rápida, tipo e geometria do equipamento de floculação e qualidade da água bruta.

O desempenho das unidades de mistura rápida e de floculação influi na qualidade da água clarificada e conseqüentemente, na duração da carreira de filtração e qualidade da água filtrada. $\mathrm{O}$ estabelecimento do tempo e do gradiente de velocidade de projeto da unidade de floculação depende, fundamentalmente, da qualidade da água bruta e da tecnologia de tratamento utilizada na ETA, conforme Pádua (1994).

Nas ETAs, a floculação pode ser realizada em unidades hidráulicas ou mecanizadas, considerando-se que cada uma apresenta uma combinação de $\mathrm{G}_{\mathrm{f}}$ (gradiente de velocidade médio de floculação) e $\mathrm{T}_{\mathrm{f}}$ (tempo de floculação) no equipamento de jarteste ou de floteste que reproduz aproximadamente essa operação em escala real. Os parâmetros $\mathrm{G}_{\mathrm{f}} \mathrm{e} \mathrm{T}_{\mathrm{f}}$ dependem de vários fatores, destacando-se o mecanismo de coagulação, tipo de coagulante, qualidade da água bruta, uso de auxiliares, etc. Em geral, o valor de $\mathrm{G}_{\mathrm{f}}$ varia de 10 a $60 \mathrm{~s}^{-1}$, enquanto $T_{\mathrm{f}}$ pode resultar entre 10 e $40 \mathrm{~min}$.

O uso de modelação matemática relativa à cinética dos encontros visa estimar o desempenho da floculação, a partir dos fenômenos de agregação e ruptura, como será visto nos itens subseqüentes.

\section{Agregação e ruptura}

Após a mistura rápida, faz-se a agitação lenta, com o objetivo de proporcionar encontros e agregar as partículas menores em maiores, denominados flocos. Com o aumento do tamanho dos flocos, as forças de cisalhamento podem causar sua ruptura. A agregação e a ruptura ocor- rem simultaneamente, conduzindo a uma única condição de distribuição de tamanho de floco. Nessas condições, a cinética dos encontros deve levar em conta estes dois fenômenos.

As duas ações básicas consideradas responsáveis pela desagregação, são:

Erosão superficial de partículas primárias presentes nos flocos: é provocada pelo arraste da água atuando por intermédio das forças de cisalhamento na superfície dos flocos, quando se tem escoamento turbulento;

Fragmentação de flocos: devido às diferenças de pressão dinâmica em lados opostos dos flocos, ocorre sua deformação e posterior fragmentação.

Segundo Di Bernardo (2002), a agregação e ruptura (desagregação) durante a floculação, resulta na formação de um tamanho de floco estável definido por:

$d_{f e s}=K_{f e s}\left(G_{m e d}\right)^{k f e s}$

$\mathrm{Na}$ equação (1), $\mathrm{d}_{\mathrm{fes}}$ representa o tamanho do floco estável (cm), $\mathrm{K}_{\text {fes }}$ o coeficiente relacionado com a resistência do floco estável (cm.s $\mathrm{s}^{\text {kfes }}$ ) e $\mathrm{k}_{\mathrm{fes}}$ (coeficiente adimensional), o coeficiente que depende do modo com que ocorre a ruptura do floco e do tamanho dos turbilhóes que causam essa ruptura, ao passo que $\mathrm{G}_{\text {med }}$ é o gradiente médio de velocidade $\left(\mathrm{s}^{-1}\right)$. Quando ocorre erosão de flocos maiores que h (micro escala de turbulência de Kolmogorof $-[\mathrm{cm}]$ ), tem-se $\mathrm{k}_{\mathrm{fes}}=2 \mathrm{e}$, para flocos menores que $h$, resulta $k_{\text {fes }}=1$. Quando a ação predominante é a de fragmentação, tem-se $\mathrm{k}_{\mathrm{fes}}=0,5$ para as duas condiçôes de tamanho de flocos em relação à h. Resultados de alguns experimentos com $\mathrm{k}_{\mathrm{fes}}=1$ indicam que o tamanho máximo do floco é inversamente proporcional a $\mathrm{G}_{\text {med }}$ (Di Bernardo, 1993).

Os resultados de estudos intensivos sobre a floculação sugeriram as seguintes relações entre $\mathrm{d}_{\text {fes }}$ e $\mathrm{G}_{\text {med }}$ para a obtenção do tamanho do floco estável, com o coeficiente $\mathrm{K}_{\text {fes }}$ englobando as ações de erosão e de fragmentação.

$d_{\text {fes }} \alpha\left(G_{\text {med }}\right)^{-(0,65 a 0,76)}$ para d $_{\text {fes }} \ll \eta \eta$

$$
d_{f e s} \alpha\left(G_{\text {med }}\right)^{-(0,8 a 1,0)} \operatorname{para~d}_{\text {fes }}>\eta
$$

A taxa de produção de partículas primárias devido à ruptura dos flocos, $\mathrm{dn}^{1} / \mathrm{dt}$, pode ser expressa por:

$$
\frac{d n^{1}}{d t}=K_{B} n^{0}\left(G_{m e d}\right)^{k e s}
$$

$\mathrm{Na}$ equação (4), $\mathrm{K}_{\mathrm{B}}$ é coeficiente de ruptura $(s), n^{0}$ é o número de partículas por unidade de volume no tempo $t=0\left(1 / \mathrm{m}^{3}\right)$, e o coeficiente $\mathrm{k}_{\mathrm{es}}$ é igual a 4 para flocos com $d>\eta$, e igual a 2, para flocos com $d<\eta$.

Considerando o fenômeno de agregação, tem-se:

$$
\frac{d n}{d t}=-\frac{4 \alpha}{n} \Phi_{f} n G_{m e d}=-K_{a g} \Phi_{f} n G_{m e d}
$$

$\mathrm{Na}$ equação (5), $\mathrm{K}_{\mathrm{ag}}$ é um coeficiente empírico que depende das características químicas do sistema e físicas da mistura; é a fração volumétrica dos flocos e n é o número de partículas por unidade devolume $\left(1 / \mathrm{m}^{3}\right)$.

Fazendo-se, $K_{A}=K_{a g} \Phi_{f}$ tem-se: $\frac{d n^{1}}{d t}=-K_{A} n^{1} G_{m e d}$

Onde $\mathrm{n}^{1}$ é o número de partículas por unidade de volume no tempot $\left(1 / \mathrm{m}^{3}\right)$.

Combinando as equaçõos (4) e (6), resulta a equação geral da floculação:

$\frac{d n^{1}}{d t}=K_{B} n^{0}\left(G_{m e d}\right)^{k e s}-K_{A} n^{1} G_{\text {med }}$

Fazendo $\mathrm{k}_{\mathrm{es}}=2$, Argaman e Kaufman (1970) aplicaram a equação (7) para uma unidade de floculação constituída de m câmaras (reatores) de mistura completa $\left(\mathrm{G}_{\mathrm{f}}\right.$ constante), em série, resultando a seguinte equação:

$\frac{n_{1}^{m}}{n_{1}^{0}}=\frac{1+K_{B} G_{f}^{2} \frac{T_{d}}{m} \sum_{i=0}^{m-1}\left(1+K_{A} G_{f} \frac{T d}{m}\right)^{i}}{\left(1+K_{A} G_{f} \frac{T d}{m}\right)^{m}}$

em que:

$\mathrm{n}_{1}^{0}$ : Número de partículas primárias por unidade de volume presente no início da floculação $\left(\mathrm{m}^{-3}\right)$

$\mathrm{n}_{1}{ }^{\mathrm{m}}$ : Número de partículas primárias por unidade de volume presente na saída da m-ésima $\left(\mathrm{m}^{-3}\right)$

$\mathrm{G}_{\mathrm{f}}$ Gradiente de velocidade médio na floculação $\left(s^{-1}\right)$

m: Número de Câmaras

$\mathrm{T}_{\mathrm{d}}$ : Tempo de floculação total (s)

$\mathrm{K}_{\mathrm{A}}$ : Coeficiente de agregação

$\mathrm{K}_{\mathrm{B}}$ : Coeficiente de ruptura (s)

Argaman et al (1970) apud Libânio (1995), apresentaram, também, um modelo que contemplava a variação dos gradientes de velocidade nas diferentes câmaras de floculação em série, conforme equação (9):

$\frac{n_{1}^{i-1}}{n_{1}^{i}}=\frac{1+K_{A} G_{f} \frac{T d}{m}}{1+\frac{n_{1}^{0}}{n_{1}^{i-1}} K_{B} G_{f}^{2} \frac{T d}{m}}$ 
Em que $\frac{n_{1}^{i-1}}{n_{1}^{i}}$ é a relação entre o nú-

mero de partículas primárias (ou turbidez) efluente e afluente de câmaras de floculação em seqüência.

Os valores dos coeficientes $\mathrm{K}_{\mathrm{A}}$ e $\mathrm{K}_{\mathrm{B}}$ deveriam ser obtidos com a realização de ensaios em instalaçóes piloto de escoamento contínuo. Tal fato dificultava a utilização do modelo devido ao custo envolvido e também ao tempo relativamente longo necessário à execução dos ensaios. Assumindo que $\mathrm{K}_{\mathrm{A}}$ e $\mathrm{K}_{\mathrm{B}}$ permaneçam constantes na equação (8), para câmaras de mistura completa em série, tais coeficientes, de acordo com Bratby et al (1977), não deveriam, teoricamente, serem alterados se o número de câmaras tendesse para o infinito, ou seja, para o escoamento do tipo pistão ou reator estático.

Segundo Bratby et al (1977), a equação para descrever a cinética da floculação em reator estático é semelhante à equação (7), dada por:

em que:

$\mathrm{n}_{0}{ }^{1}$ : Número de partículas primárias por unidade de volume no tempo $t=0\left(1 / \mathrm{m}^{3}\right)$ $\mathrm{n}_{\mathrm{t}}{ }^{1}$ : Número de partículas primárias por unidade de volume no tempo $t\left(1 / \mathrm{m}^{3}\right)$ $\frac{d n^{1}}{d t}$ :Variação de partículas por unidade $d t$ de volume em relação ao tempo $\left(1 / \mathrm{sm}^{3}\right)$

Integrando-se a equação (10) e rearranjando os termos, obtém-se:

$\frac{n_{0}^{1}}{n_{T f}^{1}}=\left[\frac{K_{B}}{K_{A}} G_{f}+\left(1-\frac{K_{B}}{K_{A}} G_{f}\right) e^{-K_{A} G F T f}\right]^{-1}$

Na equação (11), $n_{\text {Tf representa o }}^{1}$ número de partículas primárias após o tempo de floculação $T_{f}$. Os coeficientes $\mathrm{K}_{\mathrm{A}}$ e $\mathrm{K}_{\mathrm{B}}$, determinados pelo uso das equaçôes (10) e (11), podem ser usados em um sistema de diversas câmaras de mistura completa em série, com valores de $\mathrm{G}_{\mathrm{f}}$ menores que $100 \mathrm{~s}^{-1}$.

Bratby et al. (1977) estipularam que o tempo de sedimentação adotado no ensaio deveria ser relativamente longo, para que o sobrenadante apresentasse somente partículas primárias e, também, para que fosse possível utilizar os valores da turbidez remanescente para relacionálos ao número de partículas primárias no sobrenadante.

Pádua (1994) contestou esta metodologia ao questionar a validade dos dados encontrados, pois com um tempo de sedimentação longo, tem-se uma velo- cidade de sedimentação correspondente muito baixa, diferente do que realmente acontece nas estaçōes de tratamento de água, nas quais se tem valores de velocidade de sedimentação nos decantadores da ordem de 1 a $5 \mathrm{~cm} / \mathrm{min}$.

Assumindo o número de partículas primárias igual à turbidez remanescente, Bratby et al (1977) integraram a equação (10), resultando:

$\frac{N_{0}}{N_{1}}=\left[\frac{K_{B}}{K_{A}} G_{f}+\left(1-\frac{K_{B}}{K_{A}} G_{f}\right) e^{-K_{A} G F I f}\right]^{-1}$

em que:

$\mathrm{N}_{\mathrm{o}}$ : Turbidez inicial do sobrenadante (uT) $\mathrm{N}_{1}^{\circ}$ : Turbidez final do sobrenadante após tempo de sedimentação longo (uT)

\section{Determinação dos coeficientes de agregação e ruptura no equipamento de jarteste}

Para a determinação dos coeficientes de agregação e ruptura no equipamento jarteste, são realizados ensaios de coagulação, floculação e sedimentação (tempo de repouso maior ou igual a $2 \mathrm{~h}$ ) para condiçôes otimizadas de mistura rápida, junto com diferentes tempos de agitação e de gradiente de velocidade de floculação, e construídas figuras de $\mathrm{N}_{\mathrm{o}} / \mathrm{N}_{1}$ em função do tempo de floculação, para cada gradiente de velocidade estudado (Bratby et al, 1977).

Rearranjando a equação (12), obtém-se:

$$
K_{A}=\frac{1}{G_{f} T_{f}} \ln \left[\frac{\left(1-\frac{K_{B}}{K_{A}} G_{f}\right)}{\frac{1}{\frac{N_{0}}{N_{1}}}-\frac{K_{B}}{K_{A}} G_{f}}\right]
$$

Admitindo-se que não haja mais agregação nem desagregação de partículas primárias dos flocos, após um período de sedimentação relativamente longo nos jarros do equipamento de jarteste, a equação (10) pode ser igualada a zero, resultando (Bratby et al, 1977):

$$
\frac{K_{B}}{K_{A}}=\frac{1}{G_{f} \frac{N_{0}}{N_{1}}}
$$

A partir da porção horizontal da curva de melhor ajuste de todos os dados experimentais (os ensaios devem ser conduzidos até que uma porção horizontal significativa seja produzida), tem-se o valor de $\mathrm{N}_{0} / \mathrm{N}$ para cada valor de $\mathrm{G}_{\mathrm{f}}$, obtendose os valores de $\mathrm{K}_{\mathrm{B}} / \mathrm{K}_{\mathrm{A}}$.

Utilizando a equação (13) e com os valores de $K_{B} / K_{A}$ para cada $G_{f}$ (eq. 14), calcula-se e $\mathrm{K}_{\mathrm{A}}$ e $\mathrm{K}_{\mathrm{B}}$. Constrói-se então, uma curva na qual são plotados os valores de $K_{B}$ em ordenadas e $\ln \left(G_{\mathrm{f}}\right)$ em abscissas. Segundo Bratby (1981), o valor de $K_{B}$ para qualquer valor de $\mathrm{G}_{\mathrm{f}}$ é dado por uma equação do seguinte tipo:

$K_{B}=k_{1 b} \ln G_{f}+k_{2 b}$

em que:

$k_{1 b}, k_{2 b}$ : coeficientes adimensionais inerentes à água em estudo.

Considerando os aspectos relacionados aos coeficientes de agregação e de ruptura, Brito (1998) estudou dois métodos para determinar $\mathrm{K}_{A}$ e $\mathrm{K}_{B}$, a partir de dados de turbidez e do número de partículas primarias remanescentes, considerando o efeito da velocidade de sedimentação. Os métodos utilizados foram: Equação de Agregação e Ruptura - MEAR (modificação do método de Bratby, 1981) e Primeira Derivada Parcial em Relação ao Gradiente de Velocidade na Floculação-MPDPG.

O método MEAR considera a relação máxima de $\mathrm{N}_{0} / \mathrm{N}_{1}$ para cada valor de $G_{f}$, equivalente ao menor tempo de floculação em que aquela eficiência máxima é obtida. Neste método são utilizadas as equações (12) a (15), considerando-se variação nos valores dos coeficientes $\mathrm{K}_{\mathrm{A}} \mathrm{e}$ $K_{B}$ para diferentes velocidades de sedimentação e de $\mathrm{G}_{\mathrm{f}}$ como será visto posteriormente.

\section{Escalonamento do gradiente de velocidade médio na floculação}

O escalonamento consiste na aplicação de gradiente de velocidade médio maior nas primeiras câmaras de floculação, com o intuito de aumentar o número de contatos entre as partículas desestabilizadas, diminuindo-se o gradiente nas câmaras subseqüientes para evitar o rompimento acentuado dos flocos formados.

Alguns autores relatam a realização do escalonamento do gradiente de velocidade médio em ETAs de uma forma intuitiva, porém, sem demonstrar matematicamente como chegaram aos valores de $G_{f}$ nas câmaras intermediárias. Esse método intuitivo pode conduzir a um bom desempenho, inclusive melhora da eficiência, comparando-se com unidades que funcionam com $\mathrm{G}_{\mathrm{f}}$ constante, no 
entanto, não é possível garantir que os gradientes adotados forneçam a melhor eficiência da floculação.

A fim de se chegar a resultados que forneçam o gradiente ótimo para cada câmara da unidade de floculação, métodos para realização do escalonamento do gradiente de velocidade médio foram desenvolvidos por diferentes pesquisadores, sendo que Pádua (1994) propôs um método que tem sido usado com sucesso.

A partir dos resultados dos ensaios de coagulação, floculação e sedimentação em equipamento de jarteste, sob diferentes valores de gradientes e tempos de agitação, é possível determinar o gradiente de velocidade médio nas unidades de floculação de escoamento contínuo (Pádua, 1994).

\section{METODOLOGIA}

Os dados experimentais utilizados para o desenvolvimento da metodologia proposta no presente trabalho foram obtidos por Brito (1998), que estudou água com as seguintes características: $\mathrm{pH}$ de $7,7 \pm 0,2$, turbidez de $27 \pm 1 \mathrm{uT}$, cor aparente entre 200 e $220 \mathrm{uC}$, cor verdadeira na faixa de 15 a $25 \mathrm{uC}$, alcalinidade de $23 \pm 1 \mathrm{mg} / \mathrm{L}$ de $\mathrm{CaCO}_{3}$; condutividade de $46 \pm 0,5 \mu \mathrm{S} / \mathrm{cm}$ e dureza de $13 \pm 1 \mathrm{mg} / \mathrm{L} \mathrm{de} \mathrm{CaCO}_{3}$.

$\mathrm{Na}$ Tabela 1, encontra-se o resumo dos resultados, obtidos por Brito (1998), dos parâmetros ótimos de coagulação e floculação, usando cloreto férrico como coagulante no mecanismo de varredura, com dosagem de $25 \mathrm{mg} / \mathrm{L}$, para velocidades de sedimentação de 2,5 e $5,0 \mathrm{~cm} / \mathrm{min}$.

Foi determinado o gradiente de velocidade médio em unidades de floculação em série, aplicando a metodologia do escalonamento do gradiente, desenvolvida por Pádua, (1994) para 2, 3, 4 e 5 câmaras.

Ressalta-se que, neste trabalho, optou-se por escolher a maior eficiência no menor tempo de floculação que, como se pode observar nos gráficos das Figuras 1 e 2, encontram-se na faixa de 15 a 30 minutos.

\section{RESULTADOS E DISCUSSÃO}

\section{Determinação dos coeficientes $K_{A}$ e $K_{B}$}

Para a obtenção dos valores de $\mathrm{K}_{\mathrm{A}} \mathrm{e}$ $\mathrm{K}_{\mathrm{B}}$ para cada gradiente de velocidade médio e velocidade de sedimentação,

Tabela I - Parâmetros ótimos de coagulação e floculação

\begin{tabular}{lc}
\hline \multicolumn{2}{c}{ Coagulação } \\
\hline Gradiente de velocidade médio $\left(\mathrm{s}^{-1}\right)$ a $\mathrm{V}_{\mathrm{s}}=2,5$ e $5,0, \mathrm{~cm} / \mathrm{min}$ & 400 \\
Tempo de mistura $(\mathrm{s})$ & 15 \\
& Floculação
\end{tabular}

Gradiente de velocidade médio $\left(\mathrm{s}^{-1}\right)$ a $\mathrm{V}_{\mathrm{s}}=5,0 \mathrm{~cm} / \mathrm{min}$

$20,30,40,50$ e 60

Gradiente de velocidade médio $\left(s^{-1}\right) V_{s}=2,5 \mathrm{~cm} / \mathrm{min}$

$20,30,40,50$ e 60

Tempo de floculação (min)

15 e $30 \mathrm{~min}$

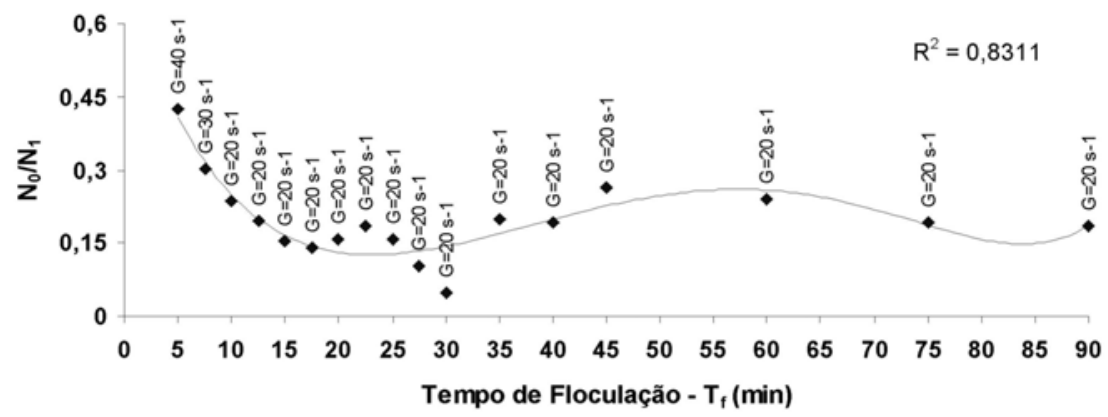

Figura I- Melhores resultados dos ensaios de otimização dos parâmetros de floculação. $V_{s}=2,5 \mathrm{~cm} / \mathrm{min}$

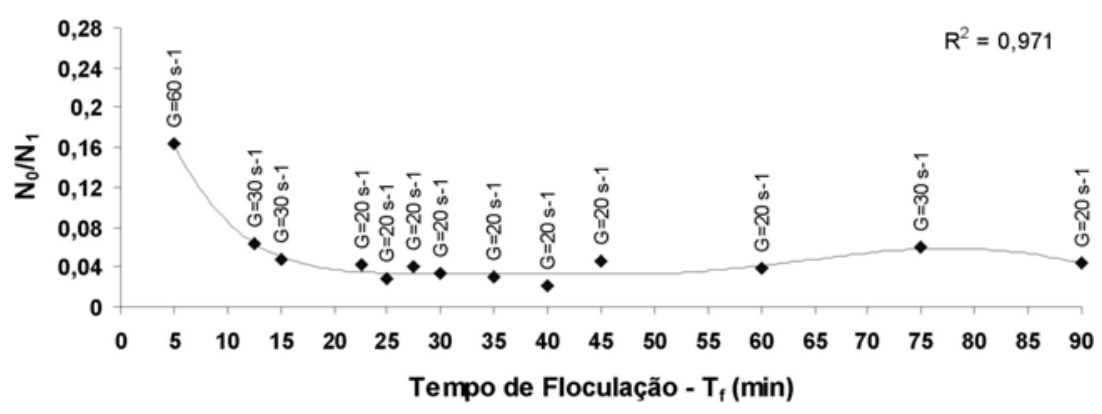

Figura 2 - Melhores resultados dos ensaios de otimização dos parâmetros de floculação. $V_{s}=5,0 \mathrm{~cm} / \mathrm{min}$

deve-se obter a maior eficiência (valores de $\mathrm{N}_{0} / \mathrm{N}_{1}$ ) para um menor tempo de floculação através da construção de gráficos de eficiência $\left(\mathrm{N}_{0} / \mathrm{N}_{1}\right)$ versus Tempo de floculação $\left(T_{\mathrm{f}}\right)$, obtidos experimentalmente nos ensaios de jarteste.

A regressão exponencial, recomendada por Bratby (1977) como regra geral, não traduz com fidedignidade a realidade dos dados experimentais, o que pode ser comprovado pelo valor de $\mathrm{R}^{2}$ das regressōes (obtidas no software excel) das curvas mostradas nas Figuras 3 e 4.

$\mathrm{O} \mathrm{R}^{2}$ é um indicador do grau de correspondência, ou coeficiente de determinação, entre os valores estimados pela regressão (linha de tendência) e os dados reais, sendo definido por:

$$
\begin{aligned}
& R^{2}=1-\frac{S S E}{S S T} \\
& S S E=\sum_{j=1}^{n}\left(Y_{j}-\overline{Y_{j}}\right)^{2} \\
& S S T=\sum_{i=j}^{n} Y_{j}-\frac{\left(Y_{j}\right)^{2}}{n}
\end{aligned}
$$

Onde $Y_{j}=$ ordenada do valor experimental, $\overline{Y_{j}}$ ordenada do valor obtido na regressão (linha de tendência) e n é o total de dados experimentais.

Quanto mais próximo de 1 for o valor de $\mathrm{R}^{2}$ mais confiável será a regressão ou a linha de tendência de ajuste dos dados experimentais. 


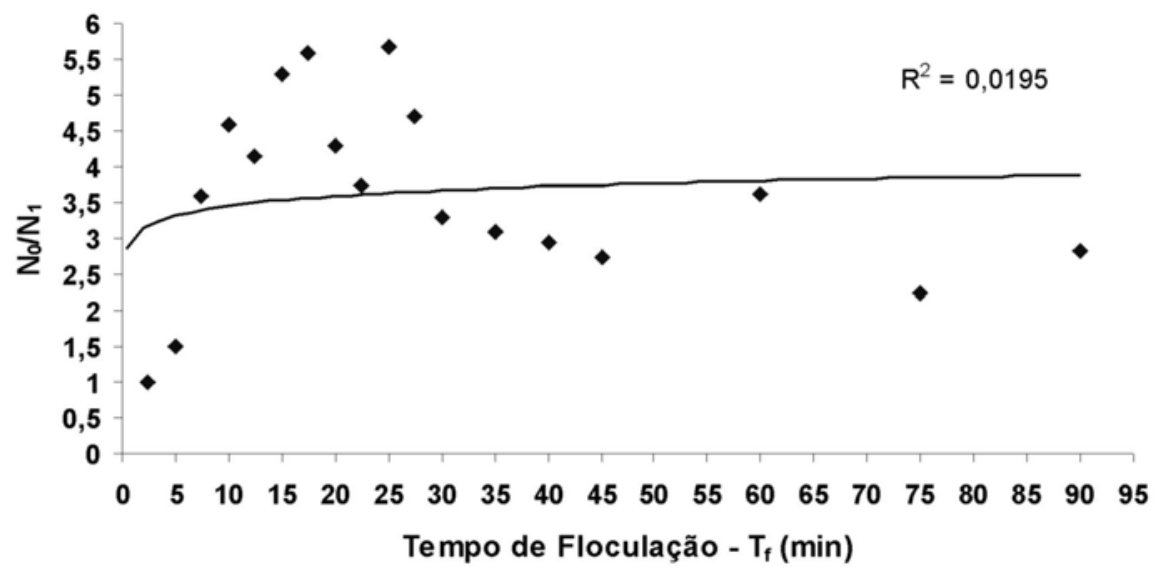

Figura 3 - Regressão exponencial para a obtenção dos coeficientes $K_{A}$ e $K_{B}$, conforme preconizada pelo método de Bratby et al (1977) - $\left(G_{f}=30 \mathrm{~s}^{-1}\right.$ e $\left.V_{s}=5,0 \mathrm{~cm} / \mathrm{min}\right)$

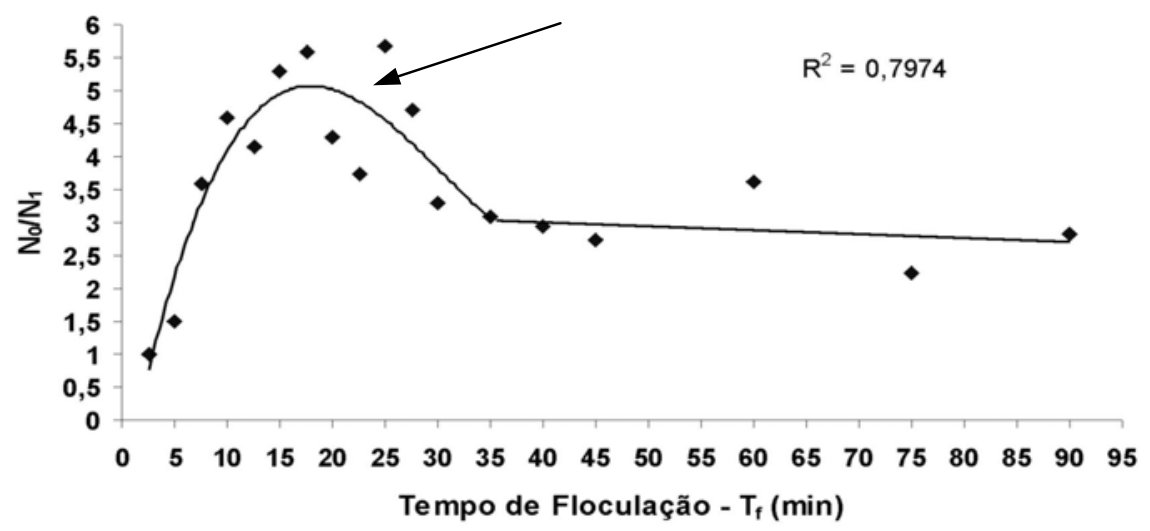

Figura 4 - Regressão polinomial para a obtenção dos coeficientes $K_{A}$ e $K_{B} \cdot\left(G_{f}=30 \mathrm{~s}\right.$ e $\left.V_{s}=5,0 \mathrm{~cm} / \mathrm{min}\right)$

No presente trabalho, considera-se, portanto, que haja uma condição específica de agregação e ruptura para cada gradiente de velocidade médio e para cada velocidade de sedimentação, o que, de fato, indica melhor aderência à curva de tendência dos dados experimentais (Figura 4).

Como pode ser visto na Figura 4, a seta indica uma região de grande intensidade de dados experimentais que são desconsiderados na regressão exponencial da Figura 3 (segundo o método de Bratby J.R., 1981).

Embora não mostrados, em praticamente todos os gráficos construídos contendogradiente de velocidade médioversus tempo de floculação utilizados neste trabalho, ocorreu a mesma região de dados indicados pela seta (Figura 4), o que justifica a utilização da regressão polinomial para a obtenção de uma região de maior eficiência.
Na obtenção dos coeficientes foram considerados valores médios de $\mathrm{K}_{A}$ e $\mathrm{K}_{B}$ para cada gradiente de velocidade médio e velocidade de sedimentação (ao invés de utilizar valores únicos, baseados em médias e em regressóes exponenciais). Foram usados os maiores valores de eficiência $\left(\mathrm{No} / \mathrm{N}_{1}\right)$ presentes nos dados experimentais, conforme seta da Figura 4. Optou-se por não utilizar a linearização de $K_{B}$, pois pode haver muita discrepância entre os valores médios dos coeficientes e os seus valores linearizados (Equação 15), conforme pode ser visto na ordem de grandeza de $\mathrm{R}^{2}$ da Figura 5.

No método MEAR, a equação (14) é resultante da equação (10) igualada a zero, ou seja, supõe-se que não haja mais variação do número de partículas primárias em relação ao tempo de floculação $\left(\mathrm{T}_{\mathrm{f}}\right)$. Matematicamente, tal fato equivale dizer que a equação (14) fornece a curva tangente ao ponto máximo da curva entre os dados experimentais, o que, de fato, pode não ser verdade, como observado por Libânio, (1995). Diversos trabalhos experimentais têm confirmado nítida redução da eficiência com o contínuo aumento do período de floculação (Di Bernardo, 2002); assim, determinar $\mathrm{K}_{\mathrm{A}} \mathrm{e}$ $K_{B}$ a partir da assintoticidade da eficiência com o tempo de floculação pode levar a resultados discrepantes e não raro, com baixas eficiências - (Libânio, 1995).

Para a obtenção dos valores de $\mathrm{K}_{\mathrm{A}} \mathrm{e}$ $\mathrm{K}_{\mathrm{B}}$ mostrados na Tabela 2, implementouse uma pequena rotina, em Excel, que objetiva a convergência entre as equações (12) e (14) através de método iterativo, disponibilizado pela ferramenta Solver do programa Microsoft Excel (Figura 6). A convergência obedeceu a limites de minimização da diferença entre os valores de $\mathrm{No} / \mathrm{N}_{1}$ das Equaçōes (12) e (14). Atribuiu-se um valor inicial de $\mathrm{K}_{\mathrm{A}}$ entre $10^{-8}$ e $10^{-20}$ e, por meio do programa solver, obtiveram-se os valores de $K_{A}$ e $K_{B}$. Deve-se ressaltar que o método consiste em uma aproximação tangencial entre a função exponencial da equação (12) e equação (14), conforme mostra o exemplo da Figura 6 (Di Bernardo et al, 2003).

\section{Escalonamento do gradiente de velocidade médio para floculadores com câmaras em série}

Foram consideradas várias situaçōes de arranjo das unidades de floculação, utilizando câmaras em série de 2 a 5 unidades com gradiente de velocidade médio escalonado, conforme Tabelas 3 e 4. Tais valores de $\mathrm{G}_{\mathrm{f}}$ foram obtidos aplicando-se a metodologia proposta por Pádua, V.L (1994), para tempos de floculação de 15 e 30 minutos.

\section{Tempo de floculação em unidades de escoamento contínuo}

Um dos maiores problemas que os engenheiros enfrentam nos projetos de dimensionamento de estaçôes de tratamento de água é a determinação do tempo de floculação em unidades de mistura completa de escoamento contínuo com câmaras em série, pelo fato dos resultados obtidos nos ensaios de jarteste, não poderem ser transpostos diretamente para estas unidades sem se considerar a influência da mudança de escala.

No ensaio em equipamento de 


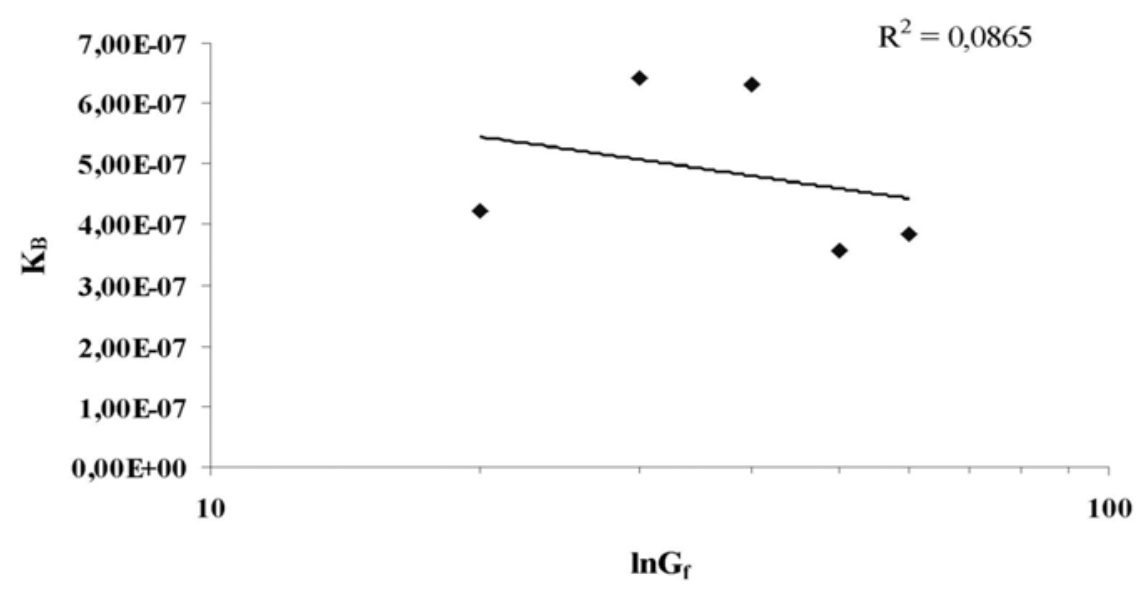

Figura 5 - Gráfico de $K_{\mathrm{B}}$ versus logaritmo natural de $G_{f}$ para a regressão linear dos valores de $K_{B}$, conforme preconizado por Bratby J.R (I98I) $-V_{s}=5,0 \mathrm{~cm} / \mathrm{min}$

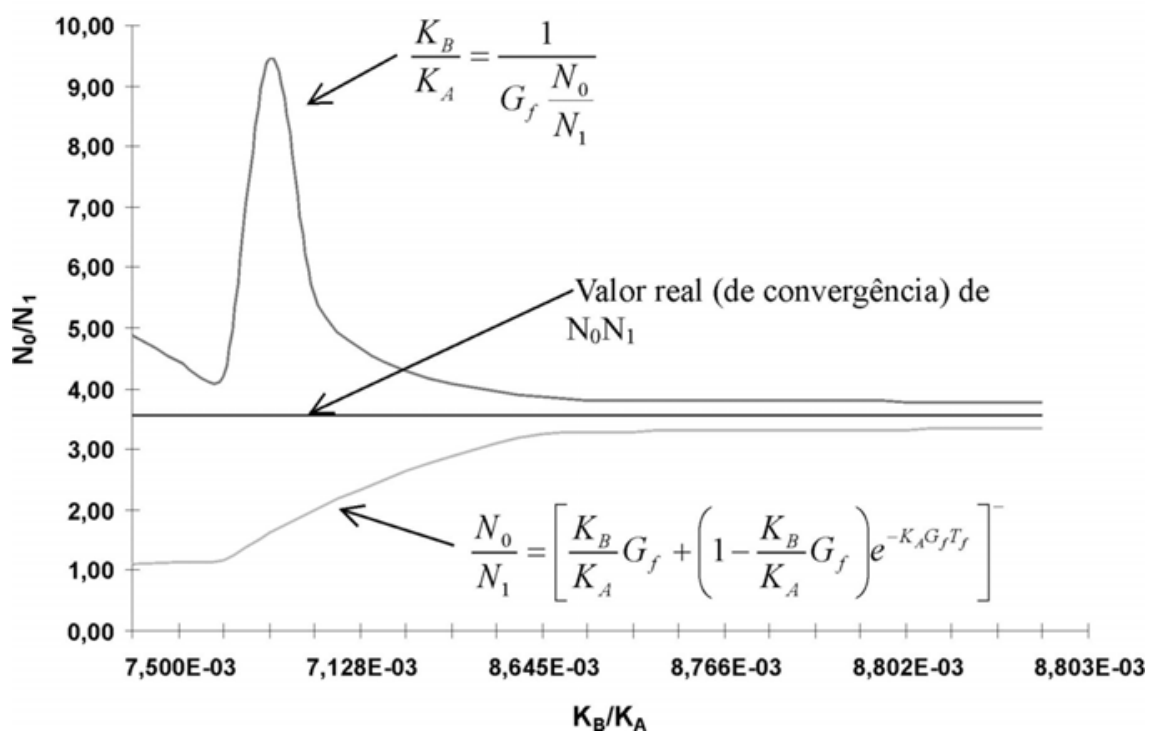

Figura 6 - Exemplo de convergência no cálculo dos valores de $K_{A}$ e $K_{B}$ jarteste, tem-se a mesma modelação aplicada ao escoamento tipo pistão ideal, de forma que os resultados obtidos neste ensaio podem ser utilizados diretamente para o projeto de unidades de floculação do tipo chicanas. Entretanto, em unidades de mistura completa em série, de escoamento continuo, os valores obtidos para o jarteste podem ser utilizados com razoável aplicabilidade, considerando-se a modelação matemática exposta neste trabalho. Deve-se ressaltar o fato de que este assunto ainda merece mais pesquisa e desenvolvimento, sobretudo na questão de se identificar um fator de escala observando-se diversos parâmetros como geometria, características do escoamento etc.

Para calcular o tempo de floculação em unidades de mistura completa em série com escoamento continuo, foram obtidos os valores de $\mathrm{N}_{0} / \mathrm{N}_{1}$ para os tempos de floculação no jarteste de 15 e $30 \mathrm{~min}$, mostrados nas Tabelas 5 a 8 .

Para quantificar a variação dos resultados transpostos de ensaio em equipamento de jarteste para reatores de mistura completa em série de escoamento continuo, foram consideradas várias situações em um arranjo de unidades de floculação utilizando com 1 a 5 câmaras em série, contemplando, tanto gradiente de velocidade médio constante (equação 8) como escalonado (equação 9), cujos resultados são apresentados nas Tabelas 5 a 8 . Foram consideradas duas velocidades de sedimentação, fixadas a partir dos dados experimentais.

Tabela 2 - Valores de K e K obtidos pelo método MEAR modificado para velocidades de 2,5 e 5,0 $\mathrm{cm} / \mathrm{m}_{\text {in }}^{\mathrm{B}}$ tempos de floculação em jarteste de 15 e 30 minutos

\begin{tabular}{ccccccccc}
\hline $\begin{array}{c}\mathrm{G} \\
{[\mathrm{s}-1]}\end{array}$ & \multicolumn{9}{c}{$\mathrm{Vs}=2,5 \mathrm{~cm} / \mathrm{min}$} \\
& \multicolumn{1}{c}{$\mathrm{K}_{\mathrm{A}}$} & $\mathrm{K}_{\mathrm{B}}$ & $\begin{array}{c}15 \mathrm{~min} \\
\mathrm{~N}_{0} \mathrm{~N}_{1}\end{array}$ & $\begin{array}{c}30 \mathrm{~min} \\
\mathrm{~N}_{0} / \mathrm{N}_{1}\end{array}$ & $\mathrm{~K}_{\mathrm{A}}$ & $\mathrm{K}_{\mathrm{B}}$ & $15 \mathrm{~min}$ & $30 \mathrm{~min}$ \\
& & & & & & $\mathrm{~N}_{0} / \mathrm{N}_{1}$ & $\mathrm{~N}_{0} / \mathrm{N}_{1}$ \\
\hline 20 & $1,83 \mathrm{E}-04$ & $1,83 \mathrm{E}-07$ & 17,69 & 46,81 & $1,90 \mathrm{E}-04$ & $4,23 \mathrm{E}-07$ & 13,25 & 22,00 \\
30 & $9,40 \mathrm{E}-05$ & $1,91 \mathrm{E}-07$ & 7,39 & 14,93 & $1,09 \mathrm{E}-04$ & $6,40 \mathrm{E}-07$ & 4,55 & 5,60 \\
40 & $6,97 \mathrm{E}-05$ & $2,33 \mathrm{E}-07$ & 4,90 & 7,17 & $5,88 \mathrm{E}-05$ & $6,31 \mathrm{E}-07$ & 2,01 & 2,29 \\
50 & $7,93 \mathrm{E}-05$ & $2,42 \mathrm{E}-07$ & 5,66 & 6,52 & $2,85 \mathrm{E}-05$ & $3,56 \mathrm{E}-07$ & 1,37 & 1,53 \\
60 & $3,68 \mathrm{E}-05$ & $2,30 \mathrm{E}-07$ & 2,17 & 2,59 & $3,18 \mathrm{E}-05$ & $3,85 \mathrm{E}-07$ & 1,29 & 1,36 \\
\hline
\end{tabular}


Di Bernardo, L.; Botary, A; Sabogal-Paz, L. P.

Tabela 3 - Valores ótimos dos gradiente de velocidade médios escalonados para 2,5 e $5,0 \mathrm{~cm} / \mathrm{min}$. ( $(T=15 \mathrm{~min}$ )

Número de Câmaras Gradientes $\left(s^{-1}\right)$

$\mathrm{V}_{\mathrm{s}}=2,5 \mathrm{~cm} / \mathrm{min} \quad \mathrm{V}_{\mathrm{s}}=5 \mathrm{~cm} / \mathrm{min}$

1

2

$40 / 30$

$30 / 20$

3

$60 / 30 / 20$

$40 / 20 / 20$

4

$60 / 30 / 30 / 20$

$50 / 30 / 20 / 20$

5
Tabela 4 - Valores ótimos dos gradiente de velocidade médios escalonados para 2,5 e $5,0 \mathrm{~cm} / \mathrm{min}$. $(T=30 \mathrm{~min})$

\begin{tabular}{ccc}
\hline Número de Câmaras & \multicolumn{2}{c}{ Gradientes $\left(\mathrm{s}^{-1}\right)$} \\
& $\mathrm{V}_{\mathrm{s}}=2,5 \mathrm{~cm} / \mathrm{min}$ & $\mathrm{V}_{\mathrm{s}}=5 \mathrm{~cm} / \mathrm{min}$ \\
\hline 1 & 30 & 20 \\
2 & $30 / 20$ & $20 / 20$ \\
3 & $30 / 20 / 20$ & $30 / 20 / 20$ \\
4 & $40 / 30 / 20 / 20$ & $30 / 20 / 20 / 20$ \\
5 & $50 / 30 / 20 / 20 / 20$ & $40 / 20 / 20 / 20 / 20$ \\
\hline
\end{tabular}

Tabela 5 - Tempo de floculação para diferentes números de câmaras em série, com gradiente de velocidade médio constante, velocidade de sedimentação de 2,5 e $5,0 \mathrm{~cm} / \mathrm{min}(T=15 \mathrm{~min})$

\begin{tabular}{ccccc}
\hline $\begin{array}{c}\text { Número } \\
\text { de câmaras }[\mathrm{m}]\end{array}$ & $\begin{array}{c}\text { Tempo total de } \\
\text { floculação para } \\
\mathrm{V}_{\mathrm{s}}=2,5 \mathrm{~cm} / \mathrm{min}, \\
\mathrm{f}=30 \mathrm{~s}^{-1}[\mathrm{~min}]\end{array}$ & {$\left[\mathrm{N}_{0} / \mathrm{N}_{1}\right]$} & $\begin{array}{c}\text { Tempo total de } \\
\text { floculação para } \\
\mathrm{V}_{\mathrm{s}}=5 \mathrm{~cm} / \mathrm{min}, \\
\mathrm{Gf}=20 \mathrm{~s}^{-1}[\mathrm{~min}]\end{array}$ & \\
\hline 1 & 68,8 & 17,69 & 130,3 & 13,25 \\
2 & 30,2 & 7,39 & 39,8 & 4,55 \\
3 & 23,6 & 4,90 & 28,0 & 2,01 \\
4 & 20,9 & 5,66 & 23,7 & 1,37 \\
5 & 19,5 & 2,17 & 21,5 & 1,29 \\
\hline
\end{tabular}

Tabela 6 - Tempo de floculação para diferentes números de câmaras em série, com gradiente de velocidade médio constante, velocidade de sedimentação de 2,5 e $5,0 \mathrm{~cm} / \mathrm{min}(T=30 \mathrm{~min})$

\begin{tabular}{ccccc}
\hline $\begin{array}{c}\text { Número } \\
\text { de câmaras }[\mathrm{m}]\end{array}$ & $\begin{array}{c}\text { Tempo total de } \\
\text { floculação para } \\
\mathrm{V}_{\mathrm{s}}=2,5 \mathrm{~cm} / \mathrm{min}, \\
\mathrm{f}=20 \mathrm{~s}^{-1}[\mathrm{~min}]\end{array}$ & {$\left[\mathrm{N}_{0} / \mathrm{N}_{1}\right]$} & $\begin{array}{c}\text { Tempo total de } \\
\text { floculação para }\end{array}$ & {$\left[\mathrm{N}_{0} / \mathrm{N}_{1}\right]$} \\
\hline 1 & 3276,6 & 46,81 & 4141,9 & 22,00 \\
$\mathrm{~V}_{\mathrm{s}}=5 \mathrm{~cm} / \mathrm{min}$, & \\
2 & 235,5 & 14,93 & 260,79 & 5,60 \\
3 & 108,9 & 7,17 & 115,8 & 2,29 \\
4 & 76,2 & 6,52 & 79,6 & 1,53 \\
5 & 62,2 & 2,59 & 64,3 & 1,36 \\
\hline
\end{tabular}


Tabela 7 - Tempo de floculação para diferentes números de câmaras em série, com gradiente de velocidade médio escalonado, velocidade de sedimentação de 2,5 e $5,0 \mathrm{~cm} / \mathrm{min}\left(T_{f}=15 \mathrm{~min}\right)$

\begin{tabular}{ccccc}
\hline $\begin{array}{c}\text { Número } \\
\text { de câmaras }[\mathrm{m}]\end{array}$ & $\begin{array}{c}\text { Tempo total de } \\
\text { floculação para } \\
\text { Vs=2,5 cm/min, } \\
{[\mathrm{min}]}\end{array}$ & $\begin{array}{c}\text { Tempo total de } \\
{\left[\mathrm{N}_{0} / \mathrm{N}_{1}\right]}\end{array}$ & $\begin{array}{c}\text { floculação para } \\
\text { Vs }=5 \mathrm{~cm} / \mathrm{min}, \\
{[\mathrm{min}]}\end{array}$ \\
\hline 1 & 68,8 & 17,69 & 130,3 & 13,25 \\
2 & 34,92 & 7,39 & 61,49 & 4,55 \\
3 & 22,61 & 4,90 & 35,95 & 2,01 \\
4 & 19,09 & 5,66 & 32,14 & 1,37 \\
5 & 13,22 & 2,17 & 22,95 & 1,29 \\
\hline
\end{tabular}

Tabela 8 - Tempo de floculação para diferentes números de câmaras em série, com gradiente de velocidade médio escalonado, velocidade de sedimentação de 2,5 e $5,0 \mathrm{~cm} / \mathrm{min}\left(T_{f}=30 \mathrm{~min}\right)$

\begin{tabular}{ccccc}
\hline $\begin{array}{c}\text { Número } \\
\text { de câmaras }[\mathrm{m}]\end{array}$ & $\begin{array}{c}\text { Tempo total de } \\
\text { floculação para } \\
\text { Vs=2,5 cm/min, } \\
{[\mathrm{min}]}\end{array}$ & $\begin{array}{c}\text { Tempo total de } \\
{\left[\mathrm{N}_{0} / \mathrm{N}_{1}\right]}\end{array}$ & $\begin{array}{c}\text { floculação para } \\
\text { Vs }=5 \mathrm{~cm} / \mathrm{min}, \\
{[\mathrm{min}]}\end{array}$ \\
\hline 1 & 3276,6 & 46,81 & 4141,9 & 22,00 \\
{$\left[\mathrm{~N}_{0} / \mathrm{N}_{1}\right]$}
\end{tabular}

Pesquisadores como Bratby, J. R (1981) afirmam que ao se realizar a floculação em câmaras de mistura completa em série, pode resultar maior eficiência global, caso o gradiente de velocidade médio de floculação decresça da primeira para a última câmara. Esta situação foi observada nos resultados obtidos neste trabalho.

A melhora na qualidade da água, quando a floculação é realizada com gradiente de velocidade médio decrescente ao longo do tempo de floculação, deve-se ao fato de que, no início, necessita-se de agitação mais intensa para aumentar as chances de contato entre as partículas desestabilizadas, para formar flocos, devendo-se reduzir a agitação posteriormente para diminuir a quebra dos flocos formados; nestas condições, o fenômeno de ruptura do floco pode ser reduzido.

Entretanto, pode ocorrer, como já mencionado, que o escalonamento nem sempre forneça ganho na eficiência (Di Bernardo et al., 2002), conforme se atesta comparando-se as Tabelas 5 e 7 para duas câmaras em serie $\left(V_{s}=5,0 \mathrm{~cm} /\right.$ min e $\mathrm{T}_{\mathrm{f}}=15 \mathrm{~min}$ ) e nas Tabelas 6 e 8 , também para o mesmo número de câmaras $\left(\mathrm{V}_{\mathrm{s}}=\right.$ $2,5 \mathrm{~cm} / \mathrm{min}$ e $\left.\mathrm{T}_{\mathrm{f}}=30 \mathrm{~min}\right)$. Porém, de umaformageral, o escalonamento tem conduzido a significativas melhoras na eficiênciaglobal dos sistemas defloculação das ETAs.

Os resultados obtidos para as velocidades de sedimentação analisadas são muito diferentes, por isso, para a realização de ensaios em reatores estáticos, devese considerar os valores de velocidade de sedimentação compatíveis com os utilizados nas estações de tratamento de água. Comprovou-se que, além da velocidade de sedimentação, recomenda-se observar, também, o tempo de floculação e sua respectiva eficiência, especialmente na etapa de estudos de concepção de projetos de floculadores.
Como observado, é desaconselhável o uso de uma única câmara de floculação no projeto de sistemas de floculação de ETAs, devido ao elevado tempo de floculação decorrente.

$\mathrm{Na}$ determinação dos valores de $\mathrm{K}_{\mathrm{A}}$ e $\mathrm{K}_{\mathrm{B}}$ neste trabalho, foi necessário estabelecer uma condição entre a diferença de $\mathrm{No} / \mathrm{N}_{\text {experimental }}$ e $\mathrm{No} / \mathrm{N}_{\text {calculada }}$ que não ultrapassasse $5 \%$ da eficiência obtida experimentalmente (jarteste). É importante observar que variações nesta condição alteram consideravelmente os valores dos coeficientes de agregação e ruptura, mudando os tempos de floculação.

\section{CONCLUSÕES E RECOMENDAÇỖES}

As principais conclusões do trabalho são as seguintes:

a) O tempo de floculação foi, de forma geral, maior para reatores de mis- 
tura completa com gradiente de velocidade médio constante, que aquele obtido com gradiente de velocidade escalonado. Isso se deve, provavelmente, ao fato de que, nestas condições, o fenômeno de ruptura de flocos nas últimas câmaras seja maior e, consequentemente, acarreta diminuição da eficiência, que se traduz pelo aumento do tempo de floculação;

b) O tempo de floculação próximo ao obtido no jarteste aconteceu quando se trabalhou com 5 câmaras em série para gradiente de velocidade médio constante e praticamente igual para gradiente de velocidade médio escalonado. Isto mostra que, aumentando-se o número de câmaras de floculação em série, o sistema trabalha mais próximo ao escoamento tipo pistão.

c) O tempo de detenção nas unidades de floculação com escoamento contínuo e câmaras com gradiente de velocidade médio escalonado ou constante, parece diminuir com o aumento do número de câmaras e tendem ao valor dos reatores estáticos.

d) Os modelos matemáticos que expressam a eficiência da floculação dificilmente podem ter seu uso generalizado para projeto e operação de estações de tratamento de água, pois foram desenvolvidos considerando-se número e concentração de partículas primárias, características específicas do escoamento e interaçōes hipotéticas entre espécies do coagulante e partículas e entre partículas desestabilizadas. Também, os modelos consideram partículas primárias remanescentes, as quais, às vezes, por serem muito pequenas, necessitam de tempos de sedimentação extremamente elevados, o que não acontece nas estaçôes de tratamento. No entanto, os modelos existentes e a metodologia apresentada permitem que a unidade de floculação de uma ETA seja projetada de forma racional, evitando-se a adoção de parâmetros que na maioria das vezes levam ao fracasso dessa operação e, como conseqüência, prejudicando o funcionamento das unidades de clarificação e filtração.

As principais recomendaçōes do trabalho em questão são:

a) Como existe uma diferença marcada entre os resultados obtidos no reator estático (jarteste) e nos reatores de escoamento continuo, não se deve utilizar diretamente o tempo de floculação obtido no ensaio de jarteste, sem considerar o escoamento do sistema e o número de unidades em série;

b) Os benefícios decorrentes da variação do gradiente de velocidade médio ao longo da unidade de floculação com escoamento contínuo são função da qualidade da água bruta, de modo que, o aumento da eficiência da floculação pode ou não ser significativa, dependendo da água estudada. Em geral, a qualidade da água de mananciais superficiais muda consideravelmente em diferentes épocas do ano, de forma que raramente são encontradas as condiçōes em que se obtiveram os dados utilizados nos modelos matemáticos de floculação. Deve-se, portanto, ressaltar o fato de que os dados deveriam advir de amostras a serem coletadas durante um período de pelo menos um ano, o que presumidamente contemplaria, ao menos, as principais variações climáticas anuais (possível padrão sazonal).

\section{AGRADECIMENTOS}

Os autores agradecem a colaboração dos seguintes pós-graduandos do Departamento de Hidráulica e Saneamento da EESC-USP: Cristiano Luchesi Niciura, Denise Conceição de Góis Santos, Francisco Gláucio Cavalcante de Souza, Mario César Cunha e Pedro Ivo de Almeida Santos.

\section{REFERÊNCIAS}

ARGAMAN, Y.; KAUFMAN, W. J. Turbulence and Flocculation. JEED-ASCE. v. 96 (5A2). p.223-241. April 1970 .

BRATBY, J. R.; MILLER, M. W.; MARAIS, G. V. R. Design of Flocculation Systems from Batch Test Data. Water S.A. v. 3 n. 4. p. 173 178. Oct. 1977

BRATBY, J. R. Interpreting Laboratory Results for the Design of Rapid Mixing and Flocculation Systems. AWWA Journal. v. 73 p: $318-325$. Jun. 1981.

BRITO, S.A. Influência da Velocidade de Sedimentação na Determinação dos Coeficientes de Agregação e Ruptura Durante a Floculação. Dissertação (mestrado) - Escola de Engenharia de São Carlos, Universidade de São Paulo, 189p. 1998.

DI BERNARDO, L. Métodos e técnicas de tratamento de água. v. 1. São Carlos. Ed. ABES, 481p. 1993.

DI BERNARDO, L., DI BERNARDO, A., CENTURIONE P. L. F. Ensaios de Tratabilidade de Água e dos Resíduos Gerados em Estaçôes de Tratamento de Água. São Carlos. Ed. RiMa, 248p. 2002.

DI BERNARDO, L., et al. Parâmetros de Projeto de Unidades de Floculação de Estações de Tratamento de Água a partir de Ensaios em Equipamentos de Jarteste. In: $22^{\circ}$ CONGRESSO BRASILEIRO DE ENGENHARIA SANITÁRIA E AMBIENTAL, Joinvile. Anais em CDRom ABES, 2003.

LIBÂNIO, M. Avaliação da Floculação em Reatores Estáticos e de Escoamento Contínuo com
Gradientes de Velocidade Constante e Variável. São Carlos. Tese (Doutorado) - Escola de Engenharia de São Carlos, Universidade de São Paulo, 136p, 1995.

PÁDUA, V. L. Metodologia para determinação dos gradientes de velocidade médios em unidades de floculação de mistura completa com câmaras em série e escoamento contínuo a partir de ensaios em reatores estáticos. São Carlos. Dissertação (Mestrado). Escola de Engenharia de São Carlos, Universidade de São Paulo, 74p. 1994.

Endereço para correspondência:

Luiz Di Bernardo

Escola de Engenharia de São

Carlos - USP

Departamento de Hidráulica e

Saneamento

Av. Trabalhador São-Carlense, 400

I 3566-590 São Carlos - SP - Brasil

Tel: (I6) 3273-95 28

E-mail: bernardo@sc.usp.brbrrrr 